\title{
PvPGIP2 Accumulation in Specific Floral Tissues But Not in the Endosperm Limits Fusarium graminearum Infection in Wheat
}

\author{
Silvio Tundo, ${ }^{1}$ Michela Janni, ${ }^{1}$ Ilaria Moscetti, ${ }^{1}$ Giulia Mandalà, ${ }^{1}$ Daniel Savatin, ${ }^{2}$ Ann Blechl, ${ }^{3}$ \\ Francesco Favaron, ${ }^{4}$ and Renato D'Ovidio'
}

\begin{abstract}
${ }^{1}$ Dipartimento di Scienze Agrarie e Forestali (DAFNE) Università della Tuscia, Via S. Camillo de Lellis snc, 01100 Viterbo, Italy; 2Dipartimento di Biologia e Biotecnologie "Charles Darwin", Sapienza Università di Roma, Piazzale Aldo Moro, 5, 00185 Roma, Italy; ${ }^{3}$ USDA-ARS, Western Regional Research Center, 800 Buchanan Street, Albany, CA 94710, U.S.A.; and ${ }^{4}$ Dipartimento Territorio e Sistemi Agro-Forestali (TeSAF), Research Group in Plant Pathology, Università di Padova, Viale dell'Università 16, 35020 Legnaro (PD), Italy
\end{abstract}

Accepted 25 September 2016.

\begin{abstract}
Fusarium head blight (FHB) caused by Fusarium graminearum is one of the most destructive fungal diseases of wheat worldwide. The pathogen infects the spike at flowering time and causes severe yield losses, deterioration of grain quality, and accumulation of mycotoxins. The understanding of the precise means of pathogen entry and colonization of floral tissue is crucial to providing effective protection against FHB. Polygalacturonase (PG) inhibiting proteins (PGIPs) are cell-wall proteins that inhibit the activity of PGs, a class of pectin-depolymerizing enzymes secreted by microbial pathogens, including Fusarium spp. The constitutive expression of a bean PGIP (PvPGIP2) limits FHB symptoms and reduces mycotoxin accumulation in wheat grain. To better understand which spike tissues play major roles in limiting $\boldsymbol{F}$. graminearum infection, we explored the use of PvPGIP2 to defend specific spike tissues. We show here that the simultaneous expression of PvPGIP2 in lemma, palea, rachis, and anthers reduced FHB symptoms caused by $\boldsymbol{F}$. graminearum compared with symptoms in infected nontransgenic plants. However, the expression of PvPGIP2 only in the endosperm did not affect FHB symptom development, indicating that once the pathogen has reached the endosperm, inhibition of the pathogen's PG activity is not effective in preventing its further spread.
\end{abstract}

Fusarium head blight (FHB) is one of the most destructive diseases of cereals grown in humid and semihumid areas (Schroeder and Christensen 1963; Steffenson 2003). Although many Fusarium species can cause FHB, depending on climatic conditions, F. graminearum Schwabe [teleomorph = Gibberella

Current address for M. Janni: IMEM-CNR, Parco Area delle Scienze 37/A, 43124 Parma, Italy.

Current address for I. Moscetti: Dipartimento di Scienze Ecologiche e Biologiche (DEB), Università della Tuscia, Largo dell'Università, 01100 Viterbo, Italy.

Corresponding author: R. D’Ovidio; E-mail: dovidio@unitus.it

*The $\boldsymbol{e}$-Xtra logo stands for "electronic extra" and indicates that five supplementary figures are published online.

(c) 2016 The American Phytopathological Society zeae (Schw.) Petch] appears to predominate (Goswami and Kistler 2004). FHB reduces grain yield and quality and causes accumulation of mycotoxins such as deoxynivalenol (DON) that make grains unsafe for human and animal consumption (Bottalico 1998; McMullen et al. 1997).

The use of $F$. graminearum strains expressing reporter genes has greatly expanded knowledge of $F$. graminearum colonization of wheat floral tissues (Brown et al. 2011; Ilgen et al. 2009). Most commonly, infection hyphae of $F$. graminearum have been seen to enter cereal floral tissues by direct penetration or through natural openings, such as stomata (Boddu et al. 2006; Bushnell et al. 2003; Pritsch et al. 2000). Histochemical and biochemical analyses have demonstrated that, in the early stages of infection, there is a protracted phase of symptomless hyphal growth in the majority of colonized tissues (Brown et al. 2011). Mycotoxin synthesis begins during the initially symptomless hyphal advance through newly colonized wheat tissue. With the onset of intracellular colonization by the pathogen, plant cells begin to die and disease symptoms become externally visible on the wheat ears (Jansen et al. 2005).

Results of several studies support the importance of the rachis tissue in the $F$. graminearum infection process. When a FHB resistance gene from Thinopyrum elongatum was introduced into hexaploid 'Chinese Spring' via substitution of the long arm of chromosome 7E (7EL), the advance of infectious hyphae from inoculated spikelets to adjacent spikelets was halted at the rachis node (Miller et al. 2011). Likewise, the major difference in the infection patterns between susceptible 'Roblin' and resistant 'Sumai 3' is that the spread of the fungus through the parenchyma and vascular tissues of the rachis is limited in the latter (Miller et al. 2004). Moreover, F. graminearum strains that do not produce DON are prevented from moving into the rachis by the development of strong cell-wall fortifications in the rachis node (Jansen et al. 2005).

The role of other floral tissues in resistance or susceptibility to $F$. graminearum infection is less well understood. Kang and Buchenauer (2000) observed greater hyphal growth on inner surfaces of lemma, glume, and palea compared with their outer surfaces. These differences were attributed to the physical and chemical features of these surfaces as well as to environmental conditions, such as humidity. Developing caryopses are highly susceptible to infection due to the absence of cell-wall thickenings in the pericarp and underlying tissues (Boenisch and Schäfer 2011). Caryopses infected with $F$. graminearum exhibit partial or complete loss of cell walls, starch granules, and of the protein matrix surrounding the starch granules. These changes 
are likely due to the activity of hydrolytic enzymes produced by the fungi growing in the infected tissues (Jackowiak et al. 2005).

The above observations lead us to hypothesize that different floral tissues may have different inherent susceptibilities to pathogen infection and host tissue colonization. In order to obtain more direct evidence of the potential of the different floral tissues to restrict the progression of Fusarium infection, we explored the use of tissue-specific expression of a bean polygalacturonase (PG) inhibiting protein (PvPGIP). PvPGIP2 is one of a group of plant defense proteins that inhibit the enzymatic activity of endo-PGs secreted by microbial pathogens to overcome the host cell-wall barrier (Kalunke et al. 2015). PvPGIP2 has been demonstrated to limit disease development, including FHB, in wheat, when expressed under control of the constitutive maize $U b i-1$ promoter, which supports accumulation of the protein in nearly all plant tissues (Ferrari et al. 2012; Janni et al. 2008). Here, we report production of transgenic bread wheat plants expressing PvPGIP2 under control of the barley Leml promoter (Skadsen et al. 2002; Somleva and Blechl 2005) or of the endosperm-specific high-molecular weight glutenin subunit (HMW-GS) Dy10 promoter (Thilmony et al. 2014). We document the tissue-specific accumulation of PvPGIP2 in lemma, palea, rachis, and anthers or in endosperm, respectively. We then compare FHB symptom development and DON accumulation among these transgenic plants and to those expressing PvPGIP2 under control of the $U b i-1$ promoter.

\section{RESULTS}

Production and characterization of wheat transgenic plants expressing PvPGIP2 under control of the Lem 1 promoter.

Two biolistic transformation experiments, called IM7 and IM9, were performed to introduce pAHC17_Lem1_PvPGIP2,

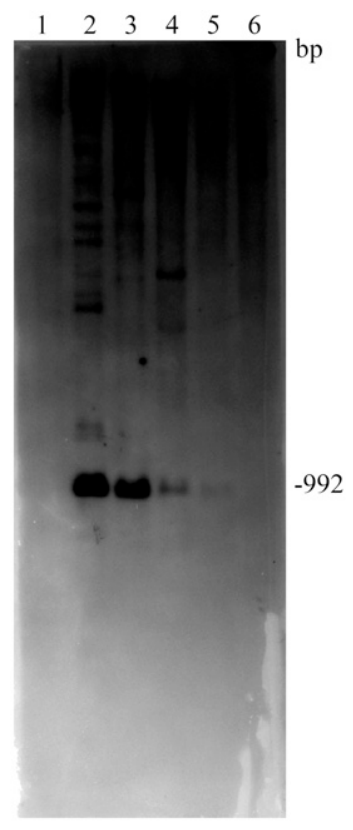

Fig. 1. Southern blots of transgenic lines expressing $P v P G I P 2$ under the Ubi-1 (J82-23a), Dy10 (J83-176), or Lem1 promoter (IM7-53 and IM9-23). Genomic DNA $(8 \mu \mathrm{g})$ from $\mathrm{T}_{3}$ Lem1 plants and $\mathrm{T}_{6}$ Ubi and Dy 10 plants was digested with HpaI (J83-176) or BamHI (J82-23a, IM7-53, and IM9-23) for the excision of the coding region of $P v P G I P 2$. The coding region of digoxigeninlabeled $P v P G I P 2$ was used as probe. 1, Triticum aestivum 'Bobwhite' digested with HpaI; 2, J82-23a; 3, J83-176; 4, IM9-23; 5, IM7-53; and 6, T. aestivum 'Bobwhite' digested with BamHI. The size and location of the PvPGIP2 fragment expected after $H p a \mathrm{I}$ or Bam $\mathrm{H} 1$ digestion is shown to the right. which contains $P v P G I P 2$ under the control of the Lem 1 promoter, and pUBI::bar, as a selectable marker into wheat 'Bobwhite'. A total of $12 \mathrm{~T}_{0}$ plants containing the PvPGIP2 gene were obtained, but only 10 of them produced seeds.

Protein extracts from floral tissues of $\mathrm{T}_{1}$ transgenic and untransformed plants at anthesis (Zadoks stage 69) were tested for PvPGIP2 inhibitor activity. Since the Lem1 promoter does not drive expression in the ovary (Somleva and Blechl 2005; confirmed below), it was removed before total protein extraction. Six transgenic lines (IM7-29, IM7-53, IM9-22, IM9-23, IM9-40, IM946) exhibited detectable inhibition activity against Fusarium phyllophilum PG (FpPG) (Supplementary Fig. S1), while the remaining four lines (IM7-28, IM9-32, IM9-48, IM9-71) did not. As expected, total protein extracts from untransformed 'Bobwhite' floral tissues did not affect FpPG activity. No inhibition activity was detected when using boiled protein extracts, thus excluding the possibility of a nonproteinaceous inhibition (data not shown). Lines IM7-53, IM9-22, and IM9-23 (hereafter Lem1 plants) showed higher inhibition activity compared with the other lines; consequently, they were selected for further characterization. No significant differences in morphology and growth were observed between the transgenic lines IM7-53 and IM9-23 up to the $\mathrm{T}_{4}$ generation and untransformed plants ( $T$. aestivum 'Bobwhite'). However, line IM9-22 showed clear phenotypic alterations starting from the $\mathrm{T}_{2}$ generation, in which it exhibited reduced height, a lower number of spikelets per spike, and smaller spikes compared with 'Bobwhite' control plants (Supplementary Fig. S2).

The integration of $P v P G I P 2$ in transgenic Lem1 plants was verified by Southern blotting. Genomic DNA of lines IM7-53 and IM9-23 digested with BamHI produced the expected 1-kb hybridization fragment containing the complete coding region of $P v P G I P 2$, whereas no hybridization signal was detected in the genomic DNA of untransformed 'Bobwhite' plants (Fig. 1). The signal intensity of the 1-kb hybridization fragment in both IM7-53 and IM9-23 was clearly lower than that in genomic DNA from the J82-23a transgenic line expressing $P v P G I P 2$ under the $U b i-1$ promoter (Ubi plants), which may indicate that the latter contains a higher number of copies of PvPGIP2 (Fig. 1).

\section{Production and characterization \\ of wheat transgenic plants expressing PvPGIP2 under control of the HMW-GS Dy10 promoter.}

A single transformation experiment called J83 was performed to introduce pJL10P5SR, which contains PvPGIP2 under control of the endosperm-specific promoter of the $H M W$ GS Dy10 gene (Thilmony et al. 2014) and pUBI::bar as selectable marker. Total protein extracts from tissues of the $T_{1}$ progeny of four independent regenerants that contained the PvPGIP2 gene (J83-159, J83-176, J83-183, and J83-200) were tested for their inhibition activity against FpPG. All the endosperm protein extracts completely inhibited the activity of FpPG, whereas none of the leaf protein extracts from the same plants showed any inhibition activity (data not shown). No significant differences in morphology and growth were observed between transgenic lines and untransformed plants. Since all four lines had strong inhibition activity, we selected the J83-176 line (hereafter Dy10 plants) for subsequent characterization.

The genomic integration of $P v P G I P 2$ in the Dy 10 plants was verified by Southern blotting. Genomic DNA of Dy10 plants digested with $H p a \mathrm{I}$ produced the expected $1-\mathrm{kb}$ fragment containing the complete coding region of $P v P G I P 2$, whereas no hybridization signal was detected in the genomic DNA of untransformed 'Bobwhite' plants (Fig. 1). The intensity of the hybridization signal in the DNA of the Dy10 plants was higher than that of the Lem1 plants (Fig. 1), which may be indicative of a higher number of copies of PvPGIP2 in line J83-176. 
PvPGIP2 driven by Lem1 accumulates at higher levels at the flowering stage.

In order to document the temporal accumulation of PvPGIP2 in the spike tissues of Lem 1 plants, the inhibition activities of transgenic IM7-53, IM9-22, and IM9-23 spikes were analyzed over the course of flowering and seed development. The amount of total protein necessary to obtain 50\% inhibition of FpPG activity was similar in all three Lem1 lines, being highest at anthesis, lowest at the early dough stage, and intermediate at the early milk and complete head emergence stages (Fig. 2A). IM922 and IM9-23 showed similar activities to one another at all stages tested (Fig. 2A). For example, $10 \pm 1.8$ and $10.9 \pm 1.4 \mu \mathrm{g}$ of total protein extract at anthesis, respectively, were necessary to inhibit by $50 \%$ the activity of FpPG (Fig. 2A). For line IM7-53, $8.3 \pm 0.8 \mu \mathrm{g}$ of total protein from anthesis inhibits $50 \%$ of FpPG activity (Fig. 2A). No inhibition activity was detected at the same growth stages in 'Bobwhite' floral tissues, even when using $20 \mu \mathrm{g}$ of total protein extract (data not shown). We also determined the temporal pattern of gene expression of $P v P G I P 2$ in floral and young seed tissues of the IM7-53 line by using quantitative reverse transcription-polymerase chain reaction (qRTPCR) to quantify relative transcript levels (Fig. 2B). PvPGIP2 transcripts were highest at the earliest stage tested (head halfemerged), 262 times higher than at the early milk stage. The decline in expression as the head emerges is precipitous, 131-fold by complete head emergence (Fig. 2B).

Both results show that the Lem1 promoter is transiently active in floral tissues and that PvPGIP2 accumulation is highest at anthesis, when the plants are most susceptible to $F$. graminearum infection.

\section{Spatial expression of PvPGIP2 driven by the Lem1 or HMW-GS Dy10 promoters and comparison between PG inhibition activities at anthesis.}

To verify the tissue specificity of $P v P G I P 2$ expression under control of the Leml or HMW-GS DylO promoters, the FpPG inhibition activities of total protein extracts from various tissues of Lem 1 or Dy 10 plants were determined. Total protein extracts $(20 \mu \mathrm{g})$ from anthers, lemma, palea, and rachis of Lem1 plants completely inhibited the activity of FpPG (Fig. 3). In contrast, no inhibition activity was detected in total protein extracts from ovaries, awns, glumes, caryopses, or leaves of Lem1 plants (Fig. 3), thus confirming the tissue-specific expression of the Leml promoter. Similar assays performed using total protein extract from corresponding tissues of Dy10 plants showed inhibition activity only in caryopses (Fig. 3), due to the accumulation of PvPGIP2 in the endosperm tissue. None of the total protein extracts from any tissue of 'Bobwhite' plants inhibited the activity of FpPG (Fig. 3).

We also compared the levels of PvPGIP2 inhibition activity in spikelets from Ubi and Lem1 plants at anthesis. Radial diffusion assays performed with various amounts of total protein extracts showed that the Ubi plants contain more than five times greater inhibition activity than Lem1 plants (Fig. 4). Indeed, $1.75 \pm 0.25 \mu \mathrm{g}$ of J83-23a total protein extract was sufficient to inhibit by $50 \%$ activity of FpPG (Fig. 4), whereas, $8.3 \pm 1.7,10 \pm$ 1.8 , and $10.9 \pm 1.4 \mu \mathrm{g}$ of total protein extracts from Lem1 lines IM753, IM9-22, and IM9-23, respectively, were necessary to inhibit by $50 \%$ activity of FpPG. No inhibition activity was detected in floral tissues of Dy10 and untransformed 'Bobwhite' plants at anthesis, even using $20 \mu \mathrm{g}$ of total protein extract (data not shown). The finding of higher inhibition activity in the Ubi plants was supported by measuring the levels of $P v P G I P 2$ transcript accumulation in the floral tissues of the Ubi and IM7-53 Lem1 plants at two developmental stages. The level of PvPGIP2 transcript was 15 -fold higher at the halfway stage of head emergence and 2,250-fold higher at anthesis in Ubi plants as compared with IM7-53 Lem1 plants.

We also compared the levels of inhibition activity of total protein extracts from the endosperm tissue of Ubi and Dy10 plants. Radial diffusion assays performed with various amounts of total protein extract found higher inhibition activity in caryopses from Dy 10 plants than in those from Ubi plants at all growth stages tested, i.e., early milk, early dough, and mature stages (Supplementary Fig. S4). In particular, $1.75 \pm 0.25 \mu \mathrm{g}$ of total protein extract from Dy10 caryopses were sufficient to inhibit by $50 \%$ FpPG activity, whereas, $8.3 \pm 1.7 \mu \mathrm{g}$ of total protein extract from caryopses of Ubi plants were needed. No inhibition activity was detected in Lem 1 and untransformed 'Bobwhite' plants, even using up to $20 \mu \mathrm{g}$ of total protein extract (data not shown).

\section{PvPGIP2 expressed in floral tissues limits FHB symptoms and DON content}

but not when expressed only in the endosperm.

Transgenic wheat plants expressing PvPGIP2 in floral tissues (Lem1 plants), endosperm tissue (Dy10 plants), and constitutively
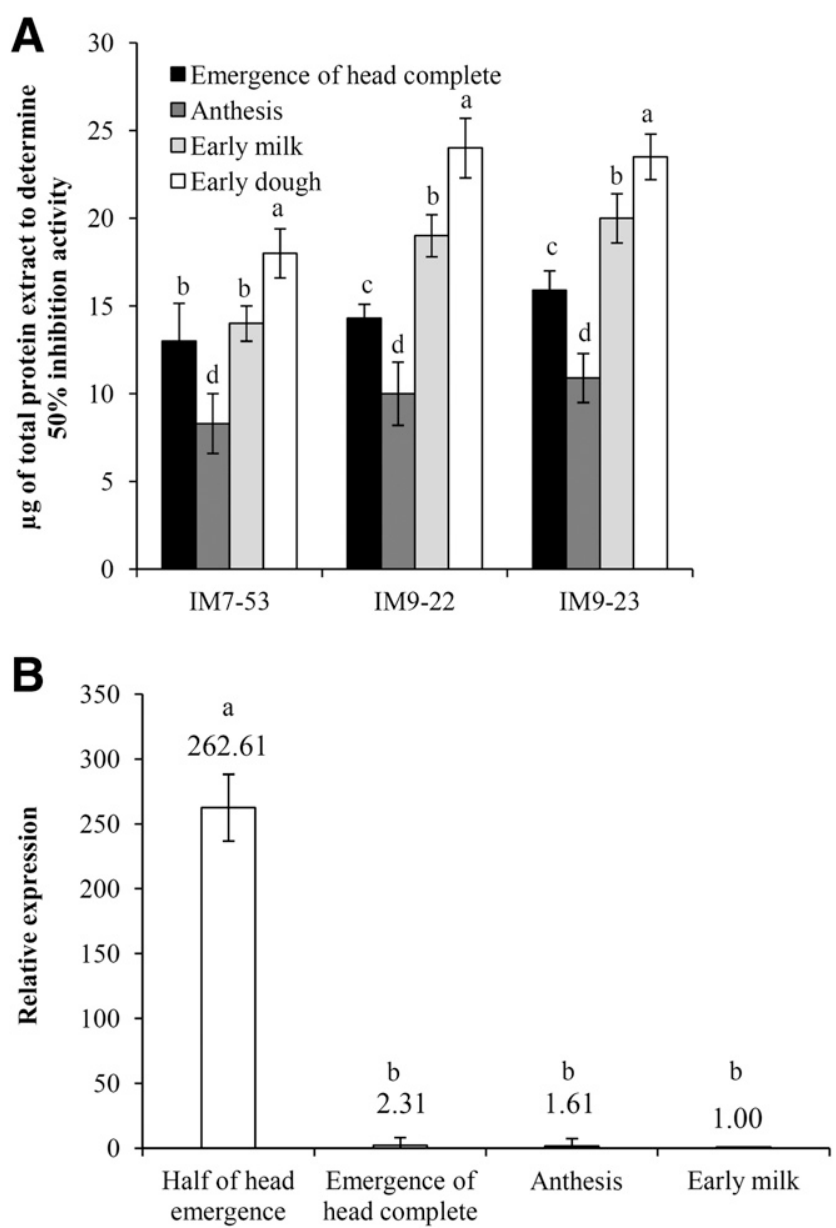

Fig. 2. Characterization of wheat transgenic plants $\left(T_{3}\right.$ generation) expressing PvPGIP2 under control of the Lem1 promoter. Bars represent the average \pm standard error of at least three biological replicates and three technical replicates. Letters above columns correspond to ranking by Tukey test at $P<0.05$. A, Inhibition activities determined by radial diffusion assay using total protein extracts of floral tissues of lines IM7-53, IM9-22, and IM9-23. Three different amounts $(10,15$, and $20 \mu \mathrm{g})$ of total protein extract from four different growth stages (emergence of head complete, anthesis, early milk, and early dough) were used against Fusarium phyllophilum polygalacturonase. No inhibition activity was detected at the same growth stages in untransformed Triticum aestivum 'Bobwhite' plants using $20 \mu \mathrm{g}$ of total protein extract (not shown). B, Expression analysis of $P v P G I P 2$ gene by quantitative reverse transcriptionpolymerase chain reaction in different spike growth stages of IM7-53 line (half of head emergence, emergence of head complete, anthesis, and early milk). Relative expression of $P v P G I P 2$ is reported as the fold increase (number above each column) of the transcript in each stage compared with the early milk stage. 
(Ubi plants) were compared for their response to $F$. graminearum infection. The three Lem1 lines expressing PvPGIP2 in floral tissues (IM7-53, IM9-22, and IM9-23) all showed reductions of FHB symptoms compared with untransformed 'Bobwhite' plants, with significantly lower percentages of bleached spikelets from 6 to 11 days postinoculation (Fig. 5). In this time range, the reduction of FHB symptoms was more than $25 \%$ for all three transgenic lines. A similarly delayed pattern of FHB development was observed in infected J82-23a plants expressing PvPGIP2 constitutively (Fig. 5), in agreement with previous results. In contrast, no difference in FHB incidence was scored between the Dy10 line J83-176 expressing PvPGIP2 only in the endosperm and untransformed 'Bobwhite' plants (Fig. 5).

In order to ascertain whether or not the reductions in visual FHB symptoms were correlated to reductions in mycotoxin accumulation in the caryopses, we quantified DON levels in the flours from Dy10, Lem1 and Ubi plants. Lem1 lines IM7-53, IM9-23, and Ubi flour showed significantly lower amounts of DON accumulation $(0.07 \pm 0.00,0.32 \pm 0.02$, and $0.12 \pm 0.01 \mu \mathrm{g}$ per milligram of flour, respectively) compared with Dy10 and 'Bobwhite' plants $(1.29 \pm 0.08$ and $0.55 \pm 0.04 \mu \mathrm{g}$ per milligram of flour, respectively).

Finally, to verify that the $\mathrm{PG}$ activity secreted by $F$. graminearum into infected caryopses of Dy10 plants was inhibited by the accumulated PvPGIP2 in this tissue, we performed agarose diffusion assays using total protein extracts from infected caryopses of Dy 10 and control 'Bobwhite' plants. PG activity was present in total protein extracts from infected 'Bobwhite' caryopses but was absent in total protein extracts from infected Dy10 caryopses (Supplementary Fig. S5). These results indicate that PvPGIP2 is a functional inhibitor of PG in wheat endosperm tissue. Moreover, total protein extracts from Dy10 caryopses completely inhibited PG activity in total protein extracts from Fusariuminfected 'Bobwhite' caryopses.

\section{DISCUSSION}

Until now, the course of $F$. graminearum colonization of wheat spikes has been investigated largely by using strains transformed with reporter genes to follow the progress of infection (Boenisch and Schäfer 2011; Jackowiak et al. 2005; Lahlali et al. 2015). These analyses highlighted differences in susceptibility to Fusarium infection among various wheat spike organs. In an attempt to obtain direct evidence of the potential of the different spikelet tissues to resist $F$. graminearum infection, we took a different approach and engineered wheat to produce the defense protein PvPGIP2 in specific tissues. The functionality of this protein is easy to assay and it has been shown to be effective in different genetically engineered plants (Kalunke et al. 2015), including against $F$. graminearum in wheat (Ferrari et al. 2012). One clear result we obtained is that expression of $P v P G I P 2$ under control of the floral-specific barley Lem-1 promoter was just as effective in reducing FHB symptoms and DON accumulation as expressing $P v P G I P 2$ under control of the constitutive $U b i-1$ promoter. The Lem-1 promoter is active in rachis, lemma, palea, and anthers and its potential use to engineer FHB resistance in wheat had been proposed by Somleva and Blechl (2005) on the basis of its capacity to drive expression in these tissues that interact with Fusarium spp. early in its colonization of the spikelet. Moreover, since the Lem-1 promoter is only transiently active in floral tissues and not active in caryopses, defense proteins synthesized under its control would not be

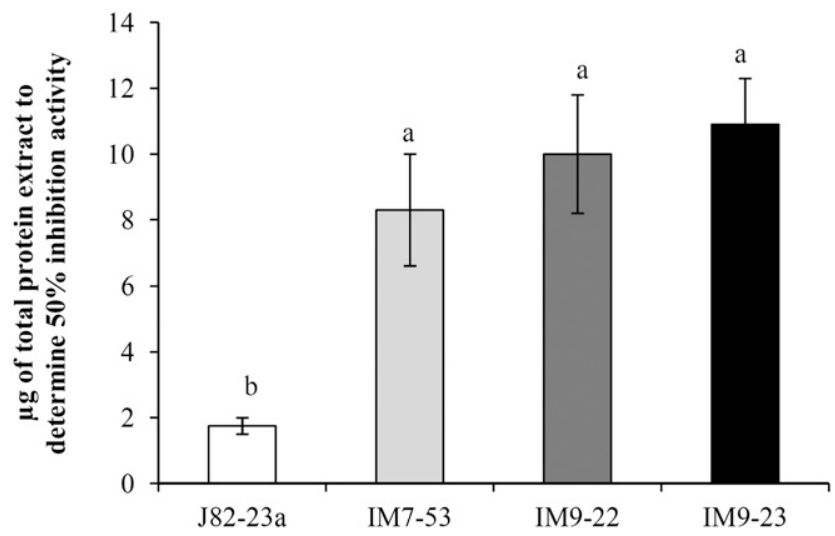

Fig. 4. Comparison between the PvPGIP2 inhibition activity of Ubi and Lem1 plants. Levels of inhibition were determined by radial diffusion assays for total protein extracts from spikelets at the flowering stages of transgenic lines J83-23a, IM7-53, IM9-22, and IM9-23. Different amounts of total protein extract against Fusarium phyllophilum polygalacturonase were used to determine $50 \%$ inhibition. No inhibition activity was detected in Dy10 and in untransformed Triticum aestivum 'Bobwhite' plants (not shown). Bars represent the average \pm standard error of at least three biological replicates and three technical replicates. Letters above columns correspond to ranking by Tukey test at $P<0.05$.

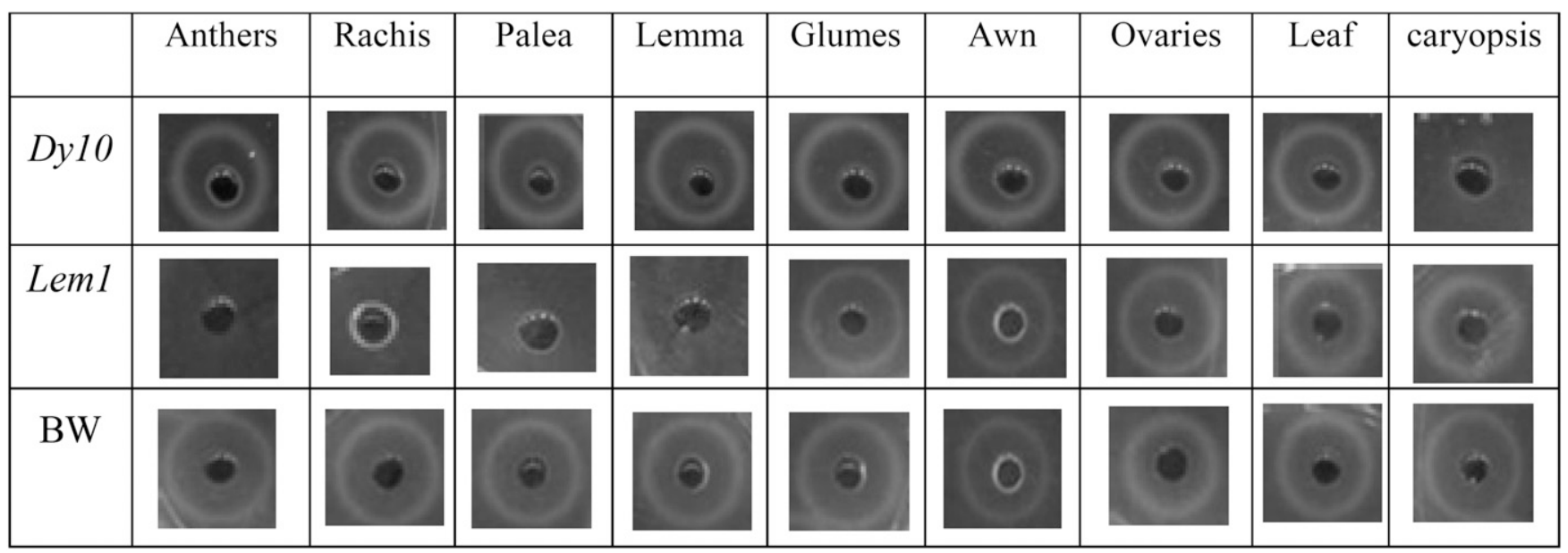

Fig. 3. Radial diffusion assay showing PvPGIP2 inhibition activity in different tissues of transgenic wheat plants expressing $P v P G I P 2$ under control of $D y 10$ promoter (top row) or Lem 1 promoter (central row). Total protein extract $(20 \mu \mathrm{g})$ was tested against $0.011 \mathrm{U}$ of Fusarium phyllophilum polygalacturonase. No inhibition activity was detected in total protein extracts of untransformed Triticum aestivum 'Bobwhite' (BW) tissues (bottom row). The absence of the halo indicates the presence of PvPGIP2 inhibition activity. Caryopses were collected at maturity, whereas the other tissues were collected at anthesis. 
made in grain. Indeed, we see no PvPGIP2 inhibition activity in the caryopses of our Lem1 transgenic plants (Fig. 3). Thus, use of the Lem-1 promoter to control synthesis of defense proteins has advantages over use of the Ubi-1 promoter in that such proteins would not be found in flour or food products derived from the transgenic plants.

In contrast, accumulation of PvPGIP2 only in the endosperm of wheat transgenic plants is not effective in combating FHB. This is not due to its lack of functionality (Supplementary Fig. S3). Nor is it due to the absence of cell-wall degrading enzymes (CWDEs) in infected wheat caryopses (Mary Wanjiru et al. 2002), whose cell walls are visibly degraded (Jackowiak et al. 2005). Rather, it may be that the large amount of starch and storage proteins in the developing endosperm makes PG and, possibly, other CWDE enzymes dispensable for the success of the pathogen in colonizing this host tissue. Thus, the inhibition of the secreted PG activity by PvPGIP2 does not produce any protective outcome in the Dy10 transgenic plants.

Our results reinforce previous observations showing the importance of lemma, palea, rachis, and anthers during $F$. graminearum infection and colonization of the host tissue. However, they do not allow us to distinguish among the importance of these floral tissues, since the Leml promoter is active in all of them. The involvement of the rachis in the spread of $F$. graminearum during infection is supported by many other studies, including one documenting the presence of peculiar xylem and phloem cell-wall structures in the rachis internodes of the resistant 'Sumai 3' as compared with the tolerant 'FL62R1' and susceptible 'Muchmore'. The 'Sumai 3' vascular structures are hypothesized to limit the growth and spread of the fungal pathogen (Lahlali et al. 2015). The same authors, using principal component analysis of Fourier transform mid infrared (FTIR) spectra, identified significant differences in absorption spectra characteristic of pectin, cellulose, and hemicellulose between infected and noninfected wheat florets of 'Sumai 3'. These could be indicative of the presence of defense compounds, such as those involved in the reinforcement of the cell walls (Lahlali et al. 2015). These results are in line with those obtained with a $F$. graminearum strain expressing green fluorescent protein under control of TRIS promoter (Ilgen et al. 2009). Strong fluorescence was detected in the rachis node during the spread of the pathogen from inoculated to uninoculated spikelets, suggesting that the rachis tissue elicits DON biosynthesis in the pathogen (Ilgen et al. 2009). These authors showed that DON is not uniformly induced during the onset of infection; it was absent from anthers, weakly induced in lemma and palea, and did not become strongly induced until the fungus reached the rachis nodes and developing kernels (Ilgen et al. 2009). Moreover, F. graminearum that do not make DON are prevented from moving into the rachis by the development of strong cell-wall fortifications in the rachis node (Jansen et al. 2005).

Here, we have shown that the level of protection in the Lem1 plants expressing PvPGIP2 is similar to that observed in transgenic wheat plants expressing PvPGIP2 constitutively. Comparable levels of resistance against $F$. graminearum have been reported in transgenic wheat plants expressing other defense genes under control of the Leml or Ubi-1 promoters (Di et al. 2010). Worth noting, the level of PvPGIP2 in floral tissues of the Lem 1 plants is significantly lower than that in the Ubi plants, suggesting that expression of defense proteins does not have to be very high if they are located in tissues critical for pathogen infection. The presence of lower but effective levels of the PvPGIP2 defense protein benefits the transgenic plant, which can save resources for other metabolic requirements.

Finally, we showed that FHB symptoms were similarly reduced in all three Lem1 lines, including IM9-22, which has a compacted spike morphology. These plants were infected using the point-inoculation method, which bypasses the compacted spike structure that is related to FHB resistance (Buerstmayr et al. 2011) in field tests. It would be interesting to repeat the resistance tests with transgenic plants infected by spraying or by wind-borne spores.

Taken all together, our results indicate that the PvPGIP2 defense of host tissues that are infected in the late stages of pathogen progression is not effective to counteract pathogen colonization. In particular, the rich reserves of starch and storage proteins in endosperm may make the activity of the pathogen PGs and possibly of the CWDEs dispensable in $F$. graminearum colonization of this tissue. However, PvPGIP2 improves wheat resistance against $F$. graminearum when it is present in tissues colonized by the pathogen in the early stages of infection, namely the lemma, palea, rachis, and anthers.

\section{MATERIALS AND METHODS}

\section{Vector DNA construction.}

The pJL10P5SR construct carrying PvPGIP2 under control of the Dy10 promoter and Dx5 terminator (Anderson et al. 1989) was obtained by using the pJL10P5 vector (Thilmony et al. 2014). HpaI sites flanking the complete coding region of $P v P G I P 2$ were generated by PCR amplification, using the forward and reverse primers (PvPGIP2HF) 5'-CAGTTAA CATGTCCTCAAGCTTAAGCAT and (PvPGIP2HR) 5' -TTGT TAACTTAAGTGCAGGCAGGAAGA, respectively, and the FastStart high fidelity PCR system (Roche Diagnostics) under conditions specified by the manufacturer. The PCR product and the pJL10P5 vector were digested with $H p a \mathrm{I}$ and were then ligated, using T4 ligase (Promega) to generate the pJL10P5SR $(9,634 \mathrm{bp})$.

The pAHC17_Lem1_PvPGIP2 construct was prepared by inserting the complete coding region of $P v P G I P 2$ into the BamHI site of pAHC17 (Christensen and Quail 1996) under control of the barley Leml promoter and NOS terminator. The barley Lem 1 promoter (1,292 bp) was cloned from the pBGS9_Lem1_Nos vector (Somleva and Blechl 2005), using primers Lem1_HindIII_F (5'-AATAAAGCTTACAGGGCGGT GCAGCTCG) and Lem1_BamHI_R (5'-AATAGGATCCCGTGC GTTGCGTGTGTGC), which added a HindIII site and a BamHI site at the $5^{\prime}$ and $3^{\prime}$ ends of the sequence, respectively. The

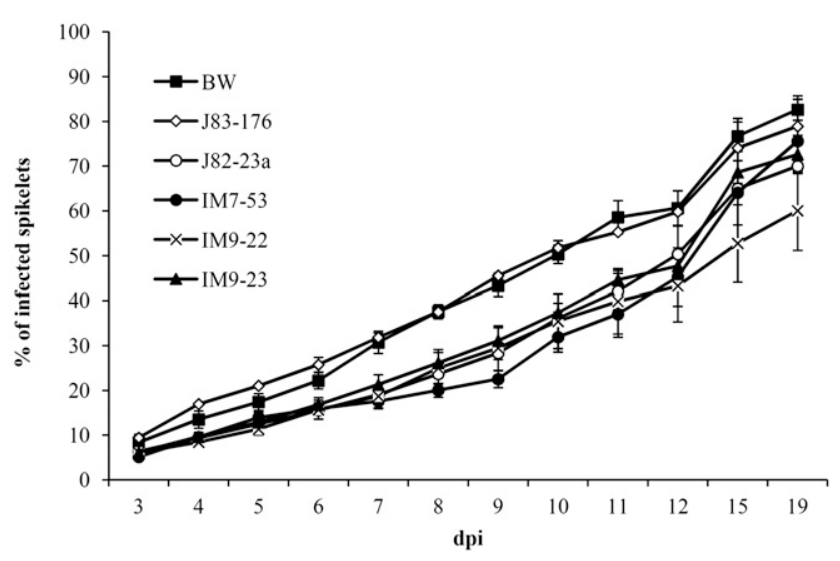

Fig. 5. Fusarium head blight (FBH) symptom development following Fusarium graminearum infection on untransformed Triticum aestivum 'Bobwhite' plants and transgenic lines expressing $P v P G I P 2$. From 6 to 11 days postinoculation, the percentage of infected spikelets in Lem1 plants (lines IM7-53, IM9-22 and IM923) and Ubi plants (line J82-23a) is significantly lower compared with untransformed T. aestivum 'Bobwhite' plants and Dy 10 plants, according to one-way analysis of variance, ranking by Tukey test at a 0.95 confidence level and a $P<0.05$ level of significance. Data represent the averages and standard errors of three experiments performed with at least 15 plants per genotype. 
amplicon was double-digested with HindIII and BamHI (Promega), following the manufacturer's protocol and were then ligated into the HindIII/BamHI sites of pAHC17 to generate the pAHC17_Lem1 construct. The complete coding region of $P v P G I P 2$ was digested from the PUBISR2 construct (Janni et al. 2008) with BamHI, following the manufacturer's protocol and, then, were ligated into the BamHI site of pAHC17_Lem1, generating pAHC17_Lem1_PvPGIP2 (4,984 bp).

The correct sequences and the insertion sites of the pJL10P5SR and pAHC17_Lem1_PvPGIP2 constructs were confirmed by nucleic acid sequencing. Both constructs were used to transform Escherichia coli DH5 $\alpha$ competent cells. The isolation of plasmid DNA was performed using commercial plasmid extraction kits (Qiagen), according to the protocols described by the manufacturer.

\section{Production and characterization}

of bread wheat transgenic plants expressing PvPGIP2.

For the wheat biolistic transformation experiments, the plasmid pUbi::bar (5,505 bp), carrying the bar gene that confers resistance to the bialaphos herbicide, was cobombarded, in 1:3 molar ratios, with the pJL10P5SR $(9,634 \mathrm{bp})$ in a single transformation experiment named J83 or with the pAHC17_Lem1_PvPGIP2 (4,984 bp) in the IM7 and IM9 transformation experiments. DNA plasmids were introduced into immature embryo-derived calli of Triticum aestivum 'Bobwhite', as described by Janni et al. (2008). In transformation experiment IM7, 1,017 immature embryos were bombarded and 10 independent $\mathrm{T}_{0}$ plants were regenerated under bialaphos selection. Three $\mathrm{T}_{0}$ plants contained the PvPGIP2 transgene, giving an overall transformation efficiency of $0.29 \%$. In transformation experiment IM9, 1,299 immature embryos were bombarded and 23 independent $T_{0}$ plants were regenerated under bialaphos selection. PCR analysis confirmed the presence of the PvPGIP2 transgene in nine of those, giving a transformation efficiency of $0.69 \%$. In transformation experiment $\mathrm{J} 83,2,967$ immature embryos were bombarded and 132 independent $\mathrm{T}_{0}$ plants were regenerated under bialaphos selection and $37 \mathrm{~T}_{0}$ lines contained the PvPGIP2 transgene, for a transformation efficiency of $1.24 \%$.

The presence of $P v P G I P 2$ in $\mathrm{T}_{0}$ J83, IM7, and IM9 bialaphosresistant plants was verified by PCR, using total DNA obtained from leaf sections of mature plants. DNA amplification was carried out according to the procedures specified for GoTaqGreen MasterMix (Promega) at an annealing temperature of $60^{\circ} \mathrm{C}$, by using the specific primer pair PvPGIP2_615F (5'-CCTCACC GGGAAGATTCCG) and PvPGIP2_853R (5'-TTAGCTGCGT CAGTCCCTGC) that produces an amplicon of $238 \mathrm{bp}$ from J83 transgenic plants, and the specific primer pair Lem1_789F (5'-ACCTTAACCTGGCGCCTTAG) and PvPGIP2_853R that produces an amplicon of 1,286 bp from IM7 and IM9 transgenic plants.

'Bobwhite' transgenic plants constitutively expressing PvPGIP2 under control of the maize ubiquitin promoter were those reported previously by Janni et al. (2008).

\section{Southern blot analysis.}

Southern hybridization was carried out on DNA from Dy10, Ubi, Lem1, and untransformed 'Bobwhite' plants (negative control) to confirm the integration of $P v P G I P 2$ and that the transgene was intact. Genomic DNA was extracted from Dy10, Ubi, and Lem 1 transgenic and wild-type plants, using $5.0 \mathrm{~g}$ of green leaf material and the method of Tai and Tanksley (1990). Genomic DNA $(8 \mu \mathrm{g})$ was digested with BamHI or HpaI restriction enzyme and were separated on $1.2 \%$ agarose gels, were transferred to positively charged nylon membranes (Roche Diagnostics), as described by Sambrook et al. (1989), and were hybridized with the complete PvPGIP2 coding region labeled with digoxigenin (digoxigenin-11-uridine-50-triphosphate;
Roche Diagnostics), following the procedure of D'Ovidio and Anderson (1994).

\section{Fungal PGs, PGIP extraction, enzymatic assays, qRT-PCR, and DON analyses.}

Fungal growth and endo-PG preparations were performed, as previously described by Caprari et al. (1996) for Fusarium phyllophilum polygalacturonase (FpPG). The crude total protein extracts were obtained from leaves of transgenic and wildtype plants, as previously described by D'Ovidio et al. (2004), and from floral tissues and caryopses at the following developmental stages: emergence of head complete (Zadoks stage 59), anthesis (Zadoks stage 69), early milk (Zadoks stage 73), and early dough (Zadoks stage 85). Ovaries or developing caryopses were removed before total protein extraction of floral tissues. Various amounts-10, 15, and $20 \mu \mathrm{g}$ - of protein extracts from each growth stage of transgenic IM7-53, IM9-22, and IM9-23 and 'Bobwhite' plants were tested by radial diffusion assays, as reported in Janni et al. (2008), against endo-FpPG. This enzyme was chosen because it is inhibited by PvPGIP2 but not by the endogenous wheat PGIPs (Janni et al. 2008).

Protein concentrations were determined with the "Coomassie plus-the better Bradford" assay kit (Pierce).

RNA extraction, genomic DNA elimination, first-strand cDNA synthesis, and qRT-PCR experiments were performed as described by Moscetti et al. (2015), with oligonucleotide primer pairs PvPGIP2_615F (5'-CCTCACCGGGAAGATTCCG) and PvPGIP2_853R (5'-TTAGCTGCGTCAGTCCCTGC) for the target gene PvPGIP2 (accession number DQ105561) and TaAct_77F (5'-TCCTGTGTTGCTGACTGAGG) and TaAct_312R (5'-GGT CCAAACGAAGGATAGCA) for the internal standard housekeeping Actin gene (accession number AB181991). Reaction conditions for qRT-PCR analysis were as follows: one cycle at $50^{\circ} \mathrm{C}$ for $2 \mathrm{~min}$ and $95^{\circ} \mathrm{C}$ for $5 \mathrm{~min}$, followed by 40 cycles at $95^{\circ} \mathrm{C}$ for $30 \mathrm{~s}, 60^{\circ} \mathrm{C}$ for $30 \mathrm{~s}$, and $72^{\circ} \mathrm{C}$ for $30 \mathrm{~s}$, and 90 cycles at $55^{\circ} \mathrm{C}$ for $10 \mathrm{~s}$, increasing the set point temperature after cycle 2 by $0.5^{\circ} \mathrm{C}$. Two biological replicates and three technical replicates were used.

For DON determinations, metabolites were extracted from $100 \mathrm{mg}$ of wheat semolina in $\mathrm{C}_{2} \mathrm{H}_{3} \mathrm{~N}: \mathrm{H}_{2} \mathrm{O} 86: 14$ on a horizontal shaker (Finepcr) for $24 \mathrm{~h}$ at $180 \mathrm{rpm}$. The extracts were centrifuged at $10,000 \times g$ for $10 \mathrm{~min}$ and DON was measured by liquid chromatography coupled to tandem mass spectrometry. The levels of DON were determined in whole flour obtained from all the caryopses collected from all plants (at least 15 plants for each sample) of a single $F$. graminearum infection experiment and the mean value was based on three technical replicates.

\section{Plant growth and infection assays.}

Seeds of transgenic and wild-type plants were surfacesterilized with sodium hypochlorite $(0.5 \% \mathrm{vol} / \mathrm{vol})$ for $20 \mathrm{~min}$ and were then rinsed thoroughly in sterile water. Seedlings were vernalized at $4^{\circ} \mathrm{C}$ for two weeks and, then, were grown in single pots at 18 to $23^{\circ} \mathrm{C}$ with a 14-h light period $\left(300 \mu \mathrm{E} \mathrm{m}^{-2} \mathrm{~s}^{-1}\right)$ to the Zadoks stage 68 . For plant infection with $F$. graminearum, the opposite central spikelets in primary spikes of transgenic and wild-type plants were point-inoculated with $F$. graminearum 3824 conidia. Procedures for the $F$. graminearum fungal cultures growth, conidia collection, infection of plants, and disease symptom analyses were carried out as described by Volpi et al. (2011). Three separate infection experiments were performed with at least 15 plants per genotype.

\section{Statistical analysis.}

Inhibition and infection data were subjected to one-way analysis of variance. When significant $F$ values were observed, 
a pairwise analysis was carried out by the Tukey honestly significant difference test (Tukey test) at 0.95 confidence level.

\section{ACKNOWLEDGMENTS}

Research was supported by the Italian Ministry of University and Research (grant Prin 2010-2011) and the Italian Ministry of Agricultural, Food and Forestry Policies (grant Progetto Internazionale di Sequenziamento del Genoma di Frumenti, Mappa fisica del Cromosoma 5A). M. Janni and S. Tundo were supported by 'Regione Lazio' (grant 'Ricercatori a Tempo Determinato'). The United States Department of Agriculture (USDA) is an equal opportunity provider and employer. Mention of a specific product name by the USDA does not constitute an endorsement and does not imply a recommendation over other suitable products.

\section{LITERATURE CITED}

Anderson, O. D., Greene, F. C., Yip, R. E., Halford, N. G., Shewry, P. R., and Malpica-Romero, J. M. 1989. Nucleotide sequences of the two highmolecular-weight glutenin genes from the D-genome of a hexaploid bread wheat, Triticum aestivum L. cv Cheyenne. Nucleic Acids Res. 17:461-462.

Boddu, J., Cho, S., Kruger, W. M., and Muehlbauer, G. J. 2006. Transcriptome analysis of the barley-Fusarium graminearum interaction. Mol. Plant-Microbe Interact. 19:407-417.

Boenisch, M. J., and Schäfer, W. 2011. Fusarium graminearum forms mycotoxin producing infection structures on wheat. BMC Plant Biol. 11:110.

Bottalico, A. 1998. Fusarium diseases of cereals: Species complex and related mycotoxin profiles in Europe. J. Plant Pathol. 80:85-103.

Brown, N.A., Bass, C., Baldwin, T.K., Chen, H., Massot, F., Carion, P.W.C., Urban, M., van de Meene, A.M.L. and Hammond-Kosack K.E. 2011. Characterisation of the Fusarium graminearum-wheat floral interaction. J. Pathog. 2011:626345.

Buerstmayr, M., Lemmens, M., Steiner, B., and Buerstmayr, H. 2011. Advanced backcross QTL mapping of resistance to Fusarium head blight and plant morphological traits in a Triticum macha $\times T$. aestivum population. Theor. Appl. Genet. 123:293-306.

Bushnell, W. R., Hazen, B. E., and Pritsch, C. 2003. Histology and physiology of Fusarium head blight. Pages 44-83 in: Fusarium Head Blight of Wheat and Barley. K. J. Leonard and W. R. Bushnell, eds. American Phytopathological Society Press, St. Paul, MN, U.S.A.

Caprari, C., Mattei, B., Basile, M. L., Salvi, G., Crescenzi, V., De Lorenzo, G., and Cervone, F. 1996. Mutagenesis of endopolygalacturonase from Fusarium moniliforme: Histidine residue 234 is critical for enzymatic and macerating activities and not for binding to polygalacturonaseinhibiting protein (PGIP). Mol. Plant-Microbe Interact 9:617-624.

Christensen, A. H., and Quail, P. H. 1996. Ubiquitin promoter-based vectors for high-level expression of selectable and/or screenable marker genes in monocotyledonous plants. Transgenic Res. 5:213-218.

Di, R., Blechl, A., Dill-Macky, R., Tortora, A., and Tumer, N. E. 2010. Expression of a truncated form of yeast ribosomal protein L3 in transgenic wheat improves resistance to Fusarium head blight. Plant Sci. 178:374-380.

D’Ovidio, R., and Anderson, O. D. 1994. PCR analysis to distinguish between alleles of a member of a multigene family correlated with wheat bread-making quality. Theor. Appl. Genet. 88:759-763.

D’Ovidio, R., Raiola, A., Capodicasa, C., Devoto, A., Pontiggia, D., Roberti, S., Galletti, R., Conti, E., O'Sullivan, D., and De Lorenzo, G. 2004. Characterization of the complex locus of bean encoding polygalacturonase-inhibiting proteins reveals subfunctionalization for defense against fungi and insects. Plant Physiol. 135:2424-2435.

Ferrari, S., Sella, L., Janni, M., De Lorenzo, G., Favaron, F., and D’Ovidio, R. 2012. Transgenic expression of polygalacturonase-inhibiting proteins in Arabidopsis and wheat increases resistance to the flower pathogen Fusarium graminearum. Plant Biol Stuttg 14 (Suppl 1):31-38.

Goswami, R. S., and Kistler, H. C. 2004. Heading for disaster: Fusarium graminearum on cereal crops. Mol. Plant Pathol. 5:515-525.

Ilgen, P., Hadeler, B., Maier, F. J., and Schäfer, W. 2009. Developing kernel and rachis node induce the trichothecene pathway of Fusarium graminearum during wheat head infection. Mol. Plant-Microbe Interact 22:899-908.

Jackowiak, H., Packa, D., Wiwart, M., and Perkowski, J. 2005. Scanning electron microscopy of Fusarium damaged kernels of spring wheat. Int. J. Food Microbiol. 98:113-123.
Janni, M., Sella, L., Favaron, F., Blechl, A. E., De Lorenzo, G., and D'Ovidio, R. 2008. The expression of a bean PGIP in transgenic wheat confers increased resistance to the fungal pathogen Bipolaris sorokiniana. Mol. Plant-Microbe Interact 21:171-177.

Jansen, C., von Wettstein, D., Schäfer, W., Kogel, K. H., Felk, A., and Maier, F. J. 2005. Infection patterns in barley and wheat spikes inoculated with wild-type and trichodiene synthase gene disrupted Fusarium graminearum. Proc. Natl. Acad. Sci. U.S.A. 102:16892-16897.

Kalunke, R. M., Tundo, S., Benedetti, M., Cervone, F., De Lorenzo, G., and D'Ovidio, R. 2015. An update on polygalacturonase-inhibiting protein (PGIP), a leucine-rich repeat protein that protects crop plants against pathogens. Front. Plant Sci. 6:146.

Kang, Z., and Buchenauer, H. 2000. Cytology and ultrastructure of the infection of wheat spikes by Fusarium culmorum. Mycol. Res. 104: 1083-1093.

Lahlali, R., Karunakaran, C., Wang, L., Willick, I., Schmidt, M., Liu, X., Borondics, F., Forseille, L., Fobert, P. R., Tanino, K., Peng, G., and Hallin, E. 2015. Synchrotron based phase contrast X-ray imaging combined with FTIR spectroscopy reveals structural and biomolecular differences in spikelets play a significant role in resistance to Fusarium in wheat. BMC Plant Biol. 15:24.

Mary Wanjiru, W., Kang, Z. S., and Buchenauer, H. 2002. Importance of cell wall degrading enzymes produced by Fusarium graminearum during infection of wheat heads. Eur. J. Plant Pathol. 108:803-810.

McMullen, M., Jones, R., and Gallenberg, D. 1997. Scab of wheat and barley: A re-emerging disease of devastating impact. Plant Dis. 81: 1340-1348.

Miller, S. S., Chabot, D. M. P., Ouellet, T., Harris, L. J., and Fedak, G. 2004. Use of a Fusarium graminearum strain transformed with green fluorescent protein to study infection in wheat (Triticum aestivum). Can. J. Plant Pathol. 26:453-463.

Miller, S. S., Watson, E. M., Lazebnik, J., Gulden, S., Balcerzak, M., Fedak, G., and Ouellet, T. 2011. Characterization of an alien source of resistance to Fusarium head blight transferred to Chinese Spring wheat. Botany 89: 301-311.

Moscetti, I., Faoro, F., Moro, S., Sabbadin, D., Sella, L., Favaron, F., and D'Ovidio, R. 2015. The xylanase inhibitor TAXI-III counteracts the necrotic activity of a Fusarium graminearum xylanase in vitro and in durum wheat transgenic plants. Mol. Plant Pathol. 16: 583-592.

Pritsch, C., Muehlbauer, G. J., Bushnell, W. R., Somers, D. A., and Vance, C. P. 2000. Fungal development and induction of defense response genes during early infection of wheat spikes by Fusarium graminearum. Mol. Plant-Microbe Interact. 13:159-169.

Sambrook, J., Fritsch, E. F., and Maniatis, T. 1989. Molecular Cloning-A Laboratory Manual, 2nd Ed. Cold Spring Harbor Laboratory Press, Cold Spring Harbor, NY, U.S.A.

Schroeder, H. W., and Christensen, J. J. 1963. Factors affecting resistance of wheat to scab caused by Gibberella zeae. Phytopathology 53: 831-838.

Skadsen, R. W., Sathish, P., Federico, M. L., Abebe, T., Fu, J., and Kaeppler, H. F. 2002. Cloning of the promoter for a novel barley gene, Lem1, and its organ-specific promotion of Gfp expression in lemma and palea. Plant Mol. Biol. 49:545-555.

Somleva, M. N., and Blechl, A. E. 2005. The barley Leml gene promoter drives expression specifically in outer floret organs at anthesis in transgenic wheat. Cereal Res. Commun. 33:665-71.

Steffenson, B. J. 2003. Fusarium head blight of barley: Impact, epidemics, management, and strategies for identifying and utilizing genetic resistance. Pages 241-295 in: Fusarium Head Blight of Wheat and Barley. K. J. Leonard, and W. R. Bushnell, eds. Vol. 10. The American Phytopathological Society, St. Paul, Minnesota.

Tai, T. H., and Tanksley, S. D. 1990. A rapid and inexpensive method for isolation of total DNA from dehydrated plant tissue. Plant Mol. Biol. Report. 8:297-303.

Thilmony, R., Guttman, M. E., Lin, J. W., and Blechl, A. E. 2014. The wheat HMW-glutenin 1Dy10 gene promoter controls endosperm expression in Brachypodium distachyon. GM Crops Food 5:36-43.

Volpi, C., Janni, M., Lionetti, V., Bellincampi, D., Favaron, F., and D'Ovidio, R. 2011. The ectopic expression of a pectin methyl esterase inhibitor increases pectin methyl esterification and limits fungal diseases in wheat. Mol. Plant-Microbe Interact 24:1012-1019. 